\title{
HIGH-THROUGHPUT SCREENING AND DYNAMIC STUDIES OF SELECTED COMPOUNDS AGAINST SARS-COV-2
}

\section{RATUL BHOWMIK ${ }^{1}$, RANAJIT NATH ${ }^{2}$, SAMEER SHARMA ${ }^{3 *}$, RATNA ROY ${ }^{4}$, RIYA BISWAS ${ }^{5}$}

\begin{abstract}
${ }^{1}$ Medicinal Chemistry and Molecular Modelling Lab, Department of Pharmaceutical Chemistry, School of Pharmaceutical Education and Research, Jamia Hamdard, New Delhi, India, 2Department of Pharmaceutics, NSHM Knowledge Campus, Kolkata-Group of Institutions, Kolkata, West Bengal, India, ${ }^{3 *}$ Department of Bioinformatics, BioNome Private Limited, Bengaluru, India, ${ }^{4}$ Department of Pharmacology, NSHM Knowledge Campus, Kolkata Group of Institutions, Kolkata, West Bengal, India, 5Department of Pharmaceutical Technology, Jadavpur University, Kolkata, West Bengal, India Email: sameer21.97@gmail.com
\end{abstract}

Received: 11 Sep 2021, Revised and Accepted: 19 Oct 2021

\begin{abstract}
Objective: This study was aimed to analyze the inhibitory effect of the drugs used in nanocarrier as well as nanoparticles formulation based drug delivery system selected from PubChem database literature against 3CLpro (3C-like protease) receptor of SARS-CoV-2 (severe acute respiratory syndrome coronavirus 2) by implementing several in silico analysis techniques.
\end{abstract}

Methods: This paper detailed a molecular docking-based virtual screening of 5240 compounds previously utilized in nanoparticle and nanocarrier drug delivery systems utilizing AutoDock Vina software on 3CL protease to discover potential inhibitors using a molecular docking technique.

Results: According to the results of the screening, the top two compounds, PubChem Id 58823276 and PubChem Id 60838 exhibited a high affinity for the 3CL protease binding region. Their binding affinities were- 9.6 and $-8.5 \mathrm{~kJ} / \mathrm{mol}$, indicating that they were tightly bound to the target receptor, respectively. These results outperformed those obtained using the co-crystallized native ligand, which exhibited a binding affinity of-7.4 kJ/mol. PubChem Id 60838, the main hit compound in terms of both binding affinity and ADMET analysis, displayed substantial deformability after MD simulation. As a result of the VS and molecular docking techniques, novel 3CL protease inhibitors from the PubChem database were discovered using the Lipinski rule of five and functional molecular contacts with the target protein, as evidenced by the findings of this work.

Conclusion: The findings suggest that the compounds discovered may represent attractive opportunities for the development of COVID-19 3CLpro inhibitors and that they need further evaluation and investigation.

Keywords: Molecular docking, Phytocompounds, Dynamic simulation, Drug discovery

(c) 2022 The Authors. Published by Innovare Academic Sciences Pvt Ltd. This is an open-access article under the CC BY license (https://creativecommons.org/licenses/by/4.0/) DOI: https://dx.doi.org/10.22159/ijap.2022v14i1.43105. Journal homepage: https://innovareacademics.in/journals/index.php/ijap

\section{INTRODUCTION}

According to scientific discovery, COVID-19 may have three phases. Certain medications are probably more effective when used independently of the other phases. The three stages are the viral early infection stage, the pulmonary stage, and the hyperinflammation stage. The world is currently experiencing a COVID-19 pandemic (caused by SARS-CoV-2) for which no effective antiviral drugs or immunizations have been developed. There are currently no target-specific drugs available for SARS-CoV-2, hence strategies such as repurposing existing treatments are being investigated rapidly [1]. As COVID-19 therapies, the drugs lopinavir and ritonavir, as well as chloroquine phosphate (commonly known as Aralen), arbidol, remdesivir, and dexamethasone (also known as Decadron), have all been given. The virus contains four non-structural proteins: papain-like (PLpro) and 3-chymotrypsin-like (3CLpro) proteases, RNA polymerase, and helicase. Both proteases (PLpro and 3CLpro) are involved in the transcription and replication stages of the virus. Because it is most closely related to viral replication, the 3CLpro type is regarded as the most important of the four types [2]. The primary protease 3CLpro of COVID19 exhibits 96 percent structural similarities to the SARS-CoV protease, according to a study. The 3CLpro enzyme is the major enzyme required for the proteolysis process. It destroys the viral polyprotein, separating it into functional components that can be used independently. Because of its crucial function in the virus life cycle, 3CLpro is an excellent target for the development of effective antiviral medications against a range of Coronaviruses [3].

As there is a crystal structure for the SARS-CoV-2 3CL Protease at the present, we used molecular docking to bind the virus using the SARS 3CL protease (PDB: 7dpv) as a Protein. 7-0-methyldihydromyricetin, on the other hand, has a covalent interaction with the SARS-CoV-2 3CL Protease. The 3CL protease, which is important for regulating numerous major activities of the virus and contains a highly retained catalytic domain from the SARS virus, is the second in silico docking model. Several of its functions include virus replication, making it a good approach for pharmaceutical research. A database of identified bioactive chemicals was tested against the SARS-CoV-2 3CL protease inhibitors' catalytic region [4].

When it comes to medication activity, molecular recognition is regarded as the most important factor. The phrase "drug action" refers to the pharmacological activity displayed by drug molecules when binding to the targeted protein and creating a stable proteinligand complex. SBVS, also known as structure-based virtual screening, aims to exploit and explore the molecular recognition between the target protein and the chosen ligand molecules to select specific molecules that show good binding affinity with the active sites of the targeted biological receptor allowing 3D structures to be inferred. The docking approach is based on identifying the optimal conformation or pose of the ligand with the receptor's specific active region. The dock score binding affinity indicates the binding relationship between the ligand molecules and the targeted protein. Based on calculated binding interactions, docking scores may also be used to predict the biological activity of ligand molecules. VS is a computational method that is frequently used to evaluate prospective drug candidates in a computer-simulated environment. In the area of novel drug discovery, virtual screening-based drug discovery is acknowledged as an effective strategy. It's being used to identify unique or prospective leads for more optimization and advancement as alternatives to therapeutically accessible medicines by identifying various molecular scaffolds that work on a target protein of interest [5]. From the previous decads, various types of drugs frequently used in nanoparticles, as well as nanocarrier drug delivery systems, were identified for performing virtual screening methodology. The purpose of this study was to use a target-based 
virtual screening approach to identify potential COVID-19 3CL protease inhibitors amongst compounds previously used in nanoparticle and nanocarrier drug delivery systems from the PubChem database, accompanied by a molecular docking process to determine novel inhibitors that could be used as potential leads for treating coronavirus-related infections.

\section{MATERIALS AND METHODS}

\section{Computer environment}

VSDK (Virtual Screening by Docking) may be done and executed on any version of Microsoft Windows or the LINUX platform. A highspeed computer machine with multiple operating systems was utilized to do virtual screening (windows, Linux). It also had a Java environment, a strong internet connection, and a stable power supply.

\section{Collection of dataset and preparation of ligand library}

An intensive literature review was used to choose various types of drugs frequently used in nanoparticles, as well as nanocarrier drug delivery systems from the PubChem database [6]. The structures of all the identified compounds were downloaded in SDF format. Using the python script, prepare ligand4. py, the substructures were then translated to pdbqt format. These structures were utilized to build a virtual library that would later be used for molecular docking and ADMET evaluation.

\section{Selection and preparation of receptor}

The chosen receptor molecule SARS-CoV-2 3CL protease (PDB-Id: 7dpv) was downloaded in PDB format from the protein data bank database [7]. After that, the protein molecule was imported into the AutoDock Tools program. To begin, the co-crystallized ligand was extracted to verify the protein. Following that, the protein was prepared by removing water molecules, eliminating unnecessary chains or heteroatoms, mending missing atoms, adding hydrogen atoms, computing charges (Kollman charges), and lastly, converting it to pdbqt format.

\section{Preparation of grid site}

After the preparation of protein, a grid box was generated with the cocrystallized ligand in the middle. During the docking process, AutoDock 4.2 was used; the center grid parameters were set to 23.2636, 18.7239, and 14.9490 for the $x^{-}, y_{-}$, and z-axes, in that order, and the Dimensions parameters were set to 22.9145, 27.3923, and 30.4039 for the $x-, y-$, and z-axes in angstrom, in that order, with a spacing of 0.375 and located at the center of the active site. The grid box dimensions were stored as config. txt files for docking with Autodock Vina. The generated protein pdbqt file was used to extract the co-crystallized ligand.

\section{Virtual screening and molecular docking}

The initial stage was to construct a database of tiny molecules by selecting a library, removing counter ions, adding hydrogen, resolving valency issues, protonation at physiological $\mathrm{pH}$, calculating 2D characteristics, converting 2D molecules to 3D, and minimizing energy. The second stage was to identify our target receptor using NMR or X-ray, with a resolution value of less than $2 \mathrm{~A}^{\circ}$. The final step was to locate the binding location of our desired receptor. The fourth step was to run the docking technique to estimate the optimal ligand confirmation at the selected receptor's binding region. The docking score or binding affinity was utilized to estimate and assess the interaction energy between our ligand and the target receptor. The sixth and last stage was to filter the docked molecules further based on ADME characteristics.

The AutoDock Vina was used to conduct the virtual screening process [8]. Protein was converted from pdb to pdbqt, and a Config. txt file was produced with all the information needed for VS utilizing ADT; other options were deemed default. The prepared protein was then docked against our prepared library set of ligands using AutoDock Vina. Perl Script was used for docking of our multiple ligands. The results were displayed in terms of binding affinity. The binding affinity represents the binding energy. The binding energy exhibits the extent of binding of the ligand molecule. For each docking experiment, a total of ten independent runs were performed. Each conformation was chosen based on its lowest binding energy. Furthermore, the best type of configuration would be the one that would bind with its target. The docking results were analyzed using Discovery Studio Biovia $2021[9,10]$.

\section{ADMET analysis}

The initially screened ligands obtained with the help of dock scores were then subjected to ADMET analysis. SwissADME and Pre-ADMET web servers were used to predict drug-likeness and ADMET properties of our ligand molecules $[11,12]$. Lipinski's rule was used to check whether the initially screened ligand molecules were suitable for docking. Ligand violating any two rules of Lipinski's was considered unsuitable for further screening. Other than Lipinski's rule, physicochemical analysis, as well as Drug-likeliness properties of all the ligand molecules, were also taken into consideration for the drug screening process. The toxicity study was examined with the help of the Pre-ADMET webserver. When it comes to PAINS (for pan assay interference compounds, which are also known as frequent hitters or promiscuous compounds), these are molecules with substructures that exhibit a potent reaction in assays regardless of the protein target while also appearing to give selective and optimizable hits. The most frequent PAINS are easily distinguished by the way they are constructed. Bioactivity screening was carried out using molinspiration software [13].

\section{Boiled-egg analysis}

For predicting blood-brain barrier permeability as well as gastrointestinal absorption of our selected phytochemicals, BOILED EGG was used. According to BOILED-Egg plot analysis, compounds found in the yellow region were considered to be having higher blood-brain barrier permeability, whereas compounds found in the white region of the plot were considered to be having higher gastrointestinal absorption properties [14]. The BOILED-Egg plot analysis was performed using the SwissADME web server [15].

\section{Molecular dynamics simulations}

The molecule with the best binding affinity along with satisfactory ADMET properties was further subjected to a molecular dynamics simulation study. Molecular Dynamics Simulation is a computerbased simulation approach used to analyze the physical motions of atoms or molecules. MD simulations can identify a few critical hydrogen bond interactions. MD simulations assist in protein docking and virtual screening advances. The iMODS server was utilized in this work to simulate molecular dynamics. The iMODS service aids in the exploration of normal mode analysis and generates accessible information about routes that may involve macromolecules or homologous structures.

For the hit chemical receptor complex, molecular dynamics simulations were also run using the Desmond program. Individually, the complex was solvated in an explicit water box of size 10 with a single-point charge (SPC) water model TIP3P with periodic boundary condition (PBC). The protein and ligand were modeled using the OPLS3e force field, and $\mathrm{Na}$ and $\mathrm{Cl}$-ions were added to make the total charge of the system neutral. Following that, the system was energy reduced for 2000 steps before a 50 ns production run. Following minimization, the complex was subjected to run at the NPT ensemble. Using the NoseHoover thermostatic algorithm and the Martina-Tobias-Klein approach, the system was gently heated to maintain a temperature of $300 \mathrm{~K}$ and pressure. To simulate long-range electrostatic interactions, the ParticleMesh Ewald (PME) approach was used with a grid spacing of 0.8. The Desmond package's Simulation Interaction Diagram tool was used to investigate the precise interactions between the ligand and protein. The data was examined in terms of protein and ligand RMSD and root mean square fluctuation (RMSF).

\section{RESULTS}

\section{Molecular docking analysis}

A total of 5240 compounds were identified from an intense literature survey from the PubChem database which had been previously used as drugs in nanoparticle or nanocarrier drug delivery systems. These compounds were then docked with the targeted receptor SARS-CoV-2 3CL protease. The native ligand of the retrieved receptor SARS-CoV-2 3CL protease was also separately docked with the receptor to compare the binding affinity of the native ligand with the hit compounds. The native ligand was observed to exhibit a docking score of-7.4 kJ/mol. Among the 5240 docked compounds, $-8.5 \mathrm{~kJ} / \mathrm{mol}$ was set as a cut-off score to initially 
screen the ligands based on binding affinity. A total of 17 compounds were screened and identified, which had a docking score of- 8.5
$\mathrm{kJ} / \mathrm{mol}$ (table 1, table 2). These 17 compounds were further subjected to ADME analysis.

Table 1: This table represents the structures, ids, and names of the initially screened 17 compounds

\begin{tabular}{|c|c|c|c|}
\hline S. No. & PubChem Id & Structure & Name \\
\hline 1 & 58823276 & & Benzoporphyrin \\
\hline 2 & 86280045 & & Phthalocyanine \\
\hline 3 & 3663 & & Hypericin \\
\hline 4 & 373075 & & Hydramycin \\
\hline 5 & 16678941 & & Lumacaftor \\
\hline 6 & 65064 & & Epigallocatechin Gallate \\
\hline 7 & 5280805 & & Rutin \\
\hline 8 & 11676786 & & $\begin{array}{l}\text { 3-(2-Cyanopropan-2-yl)-N-(4-methyl-3-((3-methyl- } \\
\text { 4-oxo-3,4-dihydroquinazolin-6- } \\
\text { yl)amino)phenyl)benzamide }\end{array}$ \\
\hline 9 & 42890 & & Idarubicin \\
\hline 10 & 60838 & & Irinotecan \\
\hline
\end{tabular}




\begin{tabular}{|c|c|c|c|}
\hline S. No. & PubChem Id & Structure & Name \\
\hline 11 & 104903 & & Tirilazad \\
\hline 12 & 2259 & & aurintricarboxylic acid \\
\hline 13 & 119373 & & Elacridar \\
\hline 14 & 443831 & & Carminomycin \\
\hline 15 & 5701999 & & Hespiridin \\
\hline 16 & 57417192 & & Natamycin \\
\hline 17 & 90813259 & & $\begin{array}{l}\text { (7-Amino-4-chloronaphthalen-1-yl) 3',6'- } \\
\text { diacetyloxy-3-oxospiro[2-benzofuran-1,9'- } \\
\text { xanthene]-5-carboxylate }\end{array}$ \\
\hline
\end{tabular}

\section{ADME and boiled-egg analysis}

The second step of the virtual screening methodology was based on ADME analysis. The compounds having a docking score of $-8.5 \mathrm{~kJ} / \mathrm{mol}$ and above were subjected to ADME analysis mainly based on Lipinski's rule, GI absorption property, and PAINS analysis. Compounds not complying with Lipinski's rules were ruled out. Compounds that have low GI absorption properties as well as exhibiting alerts in PAINS analysis were also ruled out for further screening. The ADME-based screening helped to identify 6 compounds from the initially screened 17 compounds (table 2). The initially screened 6 compounds are Pubchem Ids 58823276, 16678941, 1167686, 60838, 104903, and 119373. The screened 6 compounds were also subjected to Boiled-Egg analysis to visualize the intensity of GI absorption property. Besides this, the Boiled-Egg plot demonstrated that all the screened 6 compounds showed good gastrointestinal retention properties (fig. 1). Compound 1 represents PubChem Id 58823276, Compound 2 represents PubChem Id 16678941,
Compound 3 represents PubChem Id 1167686, Compound 4 represents PubChem Id 60838, Compound 5 represents PubChem Id 104903, and Compound 6 represents PubChem Id 119373 (fig. 1).

\section{Bioactivity analysis}

The compounds screened based on ADME analysis were further subjected to bioactivity screening. The bioactivity screening was performed on the Molinspiration webserver. The smiles of the secondarily screened 6 compounds were load on the Molinspiration webserver to check receptor specificity towards GPCR ligand, Ion Channel Modulator, Kinase Inhibitor, Nuclear Receptor Ligand, Protease Inhibitor, and Enzyme Inhibitor (table 3). The compounds showing specificity towards Enzyme Inhibitor activity were identified. This is because SARS-CoV-2 3CL protease is an enzyme. So, to act against it, the hit compounds should have enzyme inhibitory activity. The bioactivity screening identified 2 hit compounds, PubChem Ids 58823276, and 60838 from the previously screened 5 compounds. 
Table 2: Primary screening of the docked compounds based on dock score and ADME analysis. Yellow color highlighted compounds represent the screened compounds

\begin{tabular}{|c|c|c|c|c|c|c|c|}
\hline $\begin{array}{l}\text { Pubchem } \\
\text { Id }\end{array}$ & $\begin{array}{l}\text { Dock } \\
\text { score }\end{array}$ & Pains & $\begin{array}{l}\text { BBB } \\
\text { permeability }\end{array}$ & $\begin{array}{l}\text { GI } \\
\text { Absorption }\end{array}$ & $\begin{array}{l}\text { P-gp } \\
\text { substrate }\end{array}$ & LogS & Lipinski's Rule \\
\hline 58823276 & -9.6 & 0 alert & yes & high & no & -5.48 & Yes; 0 violation \\
\hline 86280045 & -9.5 & 0 alert & no & high & yes & -7.88 & No; 2 violations: $M W>500$, MLOGP $>4.15$ \\
\hline 3663 & -9.1 & 1 alert: ene_one_D & no & low & no & -6.99 & No; 2 violations: $\mathrm{MW}>500, \mathrm{NHorOH}>5$ \\
\hline 373075 & -9.1 & 1 alert: quinone_A & no & high & no & -3.22 & Yes; 0 violation \\
\hline 16678941 & -8.8 & 0 alert & no & high & yes & -5.45 & Yes; 0 violation \\
\hline 65064 & -8.8 & 1 alert: catechol_A & no & low & no & -3.56 & No; 2 violations: NorO $>10$, NHorOH>5 \\
\hline 5280805 & -8.7 & 1 alert: catechol_A & no & low & yes & -3.3 & $\begin{array}{l}\text { No; } 3 \text { violations: } \mathrm{MW}>500 \text {, NorO }>10 \text {, } \\
\mathrm{NHorOH}>5\end{array}$ \\
\hline 11676786 & -8.5 & 0 alert & no & high & no & -5.38 & Yes; 0 violation \\
\hline 42890 & -8.5 & 1 alert: quinone_A & no & low & yes & -4.1 & Yes; 0 violation \\
\hline 60838 & -8.5 & 0 alert & no & high & yes & -5.71 & Yes; 1 violation: $M W>500$ \\
\hline 104903 & -9.6 & 0 alert & yes & high & yes & -7.13 & Yes; 1 violation: $M W>500$ \\
\hline 2259 & -9.3 & 0 alert & no & low & no & -5.14 & Yes; 0 violation \\
\hline 119373 & -8.5 & 0 alert & no & high & no & -6.7 & Yes; 1 violation: $M W>500$ \\
\hline 443831 & -8.5 & 1 alert: quinone_A & no & low & yes & -4.01 & $\begin{array}{l}\text { No; } 3 \text { violations: } \mathrm{MW}>500 \text {, NorO }>10 \text {, } \\
\mathrm{NHorOH}>5\end{array}$ \\
\hline 5701999 & -8.5 & 0 alert & no & low & yes & -3.28 & $\begin{array}{l}\text { No; } 3 \text { violations: } \mathrm{MW}>500 \text {, NorO }>10 \text {, } \\
\text { NHorOH }>5\end{array}$ \\
\hline 57417192 & -8.6 & 0 alert & no & low & no & -2.92 & $\begin{array}{l}\text { No; } 3 \text { violations: } \mathrm{MW}>500 \text {, Nor } \mathrm{O}>10 \text {, } \\
\mathrm{NHorOH}>5\end{array}$ \\
\hline 90813259 & -9.1 & 0 alert & no & low & no & -7.7 & No; 2 violations: $M W>500$, MLOGP $>4.15$ \\
\hline
\end{tabular}

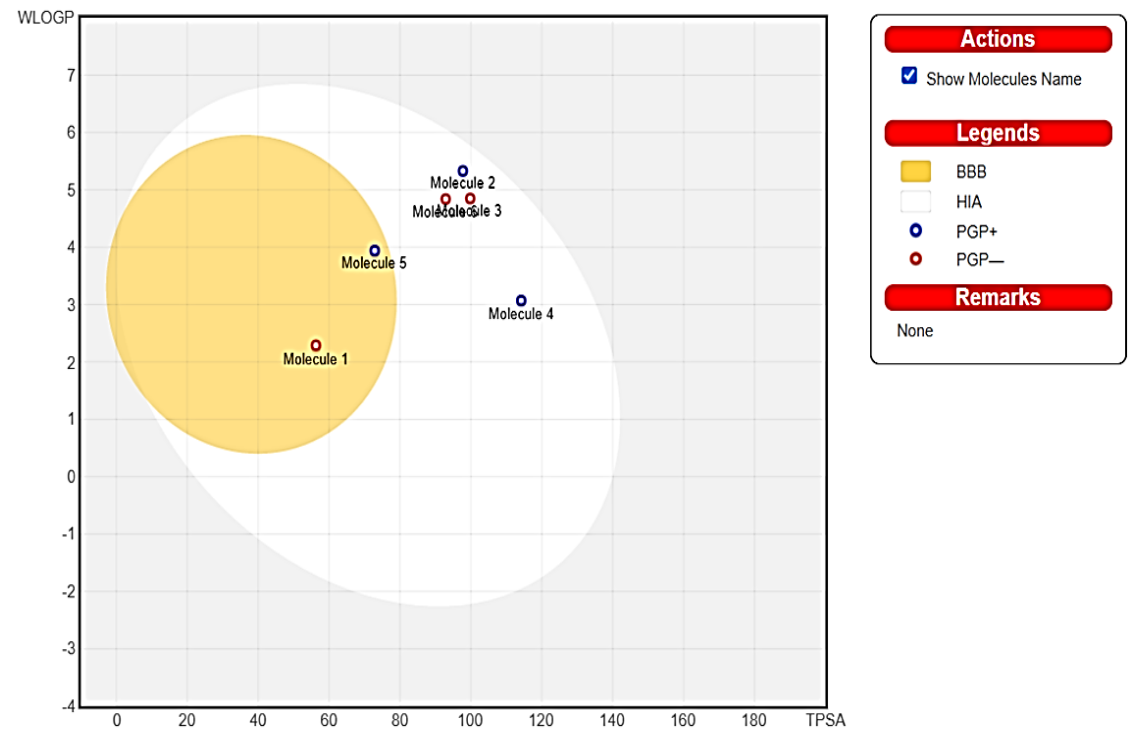

Fig. 1: This fig. represents secondarily screened 6 ligand molecules based on ADME properties. The white part of the BOILED-EGG Model represents the physicochemical space of molecules with the highest probability of being absorbed by the GI (gastrointestinal tract), while the yellow part represents the physicochemical space of molecules with the highest probability of permeating to the brain

Table 3: Secondary screening of the compounds based on bioactivity. Yellow color highlighted compounds represent the screened compounds

\begin{tabular}{|c|c|c|c|c|c|c|}
\hline $\begin{array}{l}\text { Pubchem } \\
\text { Id }\end{array}$ & $\begin{array}{l}\text { GPCR } \\
\text { ligand }\end{array}$ & $\begin{array}{l}\text { Ion channel } \\
\text { modulator }\end{array}$ & $\begin{array}{l}\text { Kinase } \\
\text { inhibitor }\end{array}$ & $\begin{array}{l}\text { Nuclear receptor } \\
\text { ligand }\end{array}$ & $\begin{array}{l}\text { Protease } \\
\text { inhibitor }\end{array}$ & $\begin{array}{l}\text { Enzyme } \\
\text { inhibitor }\end{array}$ \\
\hline 58823276 & 0.18 & 0.31 & 0.39 & -0.01 & -0.03 & 0.23 \\
\hline 16678941 & 0.21 & -0.23 & 0.12 & 0.16 & 0.1 & 0.06 \\
\hline 11676786 & -0.11 & -0.23 & 0.32 & -0.35 & -0.23 & -0.03 \\
\hline 60838 & 0.33 & -0.45 & -0.1 & -0.15 & 0.02 & 0.54 \\
\hline 104903 & 0.01 & -0.81 & -0.71 & -0.02 & 0.03 & -0.12 \\
\hline 119373 & 0.03 & -0.51 & -0.1 & -0.41 & -0.1 & -0.11 \\
\hline
\end{tabular}

\section{Toxicity analysis analysis}

The final step of the screening was toxicity prediction. The toxicity prediction was performed on the Pre-ADMET webserver. The compound with the least toxicity was identified as a hit and was further subjected to a Molecular Dynamics Simulation Study. Here compound PubChem Id 60838 showed a better toxicity profile than compound PubChem Id 58823276. Thus PubChem Id 60838 was thus identified as the main hit compound. 


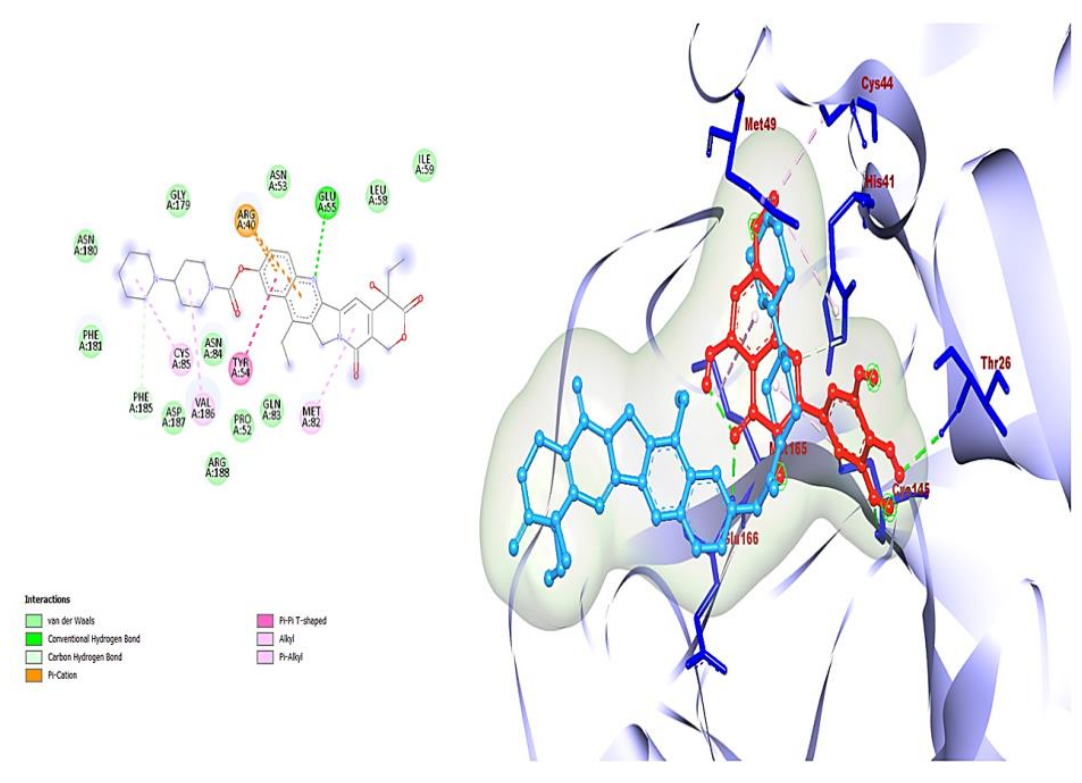

Fig. 2: Biovia discovery studio structural analysis of compound pubchem Id 60838 (sky blue) with receptor SARS-CoV-2 3CL Protease (violet) along with the native ligand (red)
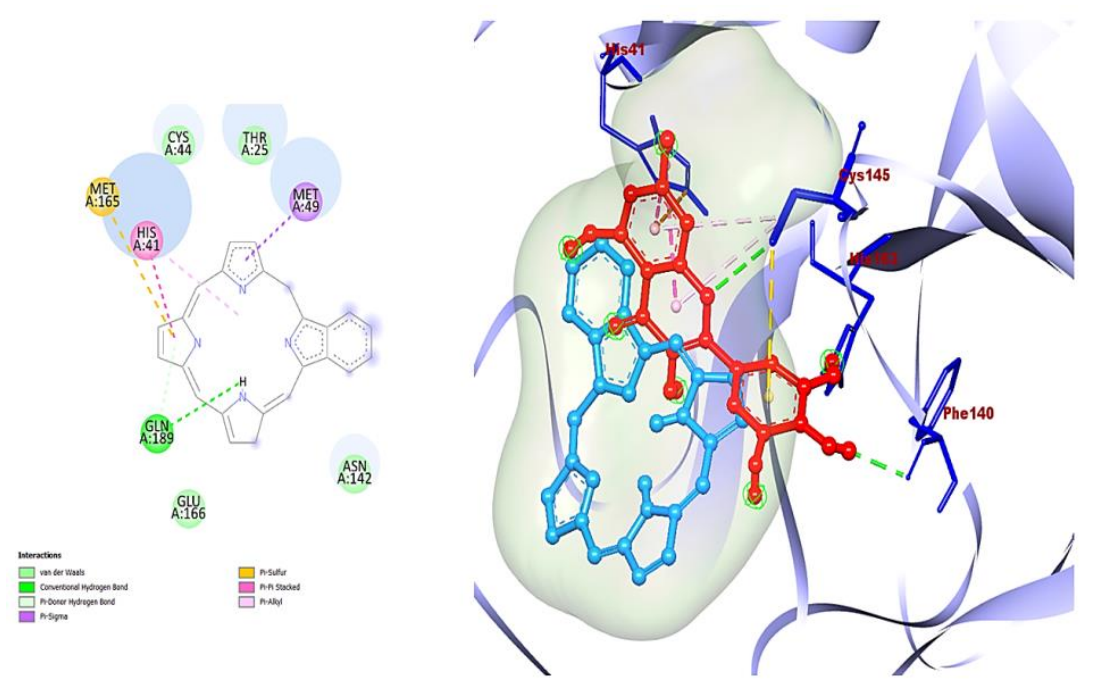

Fig. 3: Biovia discovery studio structural analysis of compound Pubchem Id 58823276 (sky blue) with receptor SARS-CoV-2 3CL Protease (violet) along with the native ligand (red)

Table 4: Final screening of the compounds based on toxicity. The yellow color highlighted compound represents the final hit compound

\begin{tabular}{lll}
\hline PubChem Id & $\mathbf{5 8 8 2 3 2 7 6}$ & $\mathbf{6 0 8 3 8}$ \\
\hline algae_at & 0.0705416 & 0.00447712 \\
Ames_test & mutagen & non-mutagen \\
Carcino_Mouse & positive & positive \\
Carcino_Rat & positive & negative \\
daphnia_at & 0.0529423 & 0.0110542 \\
hERG_inhibition & medium_risk & medium_risk \\
medaka_at & 0.00639048 & 0.000377343 \\
minnow_at & 0.0164685 & 0.00146839 \\
TA100_10RLI & positive & negative \\
TA100_NA & negative & negative \\
TA1535_10RLI & negative & negative \\
TA1535_NA & negative & negative \\
\hline
\end{tabular}

\section{Molecular dynamics simulation study}

Pubchem Id 60838 compound was identified as the best hit and was subjected to molecular dynamics simulation analysis (fig. 4). Here the docked complex of the compound Pubchem Id 60838 with receptor SARS-CoV-2 3CLpro was considered for MD simulation. Normal mode analysis mobility allows us to analyze the large-scale B-factor and mobility as well as the stability of the molecules. The IMOD server exposed the internal coordinate's analysis depending on the protein-ligand structural interactions. 


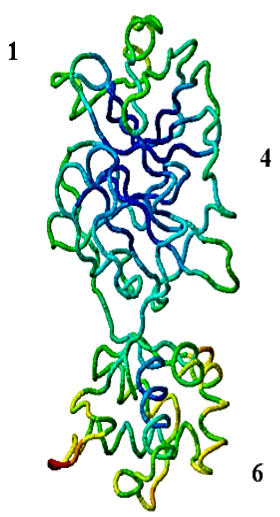

2
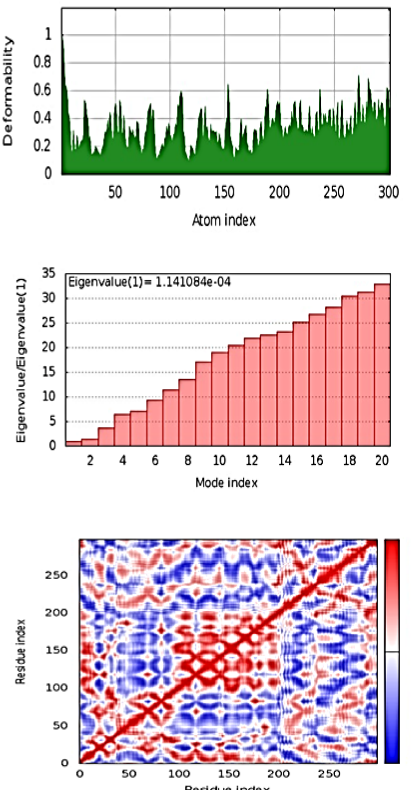

3

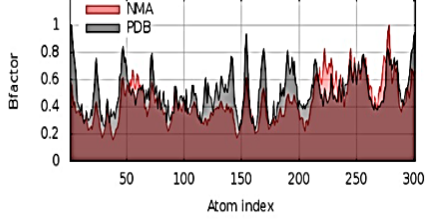

5
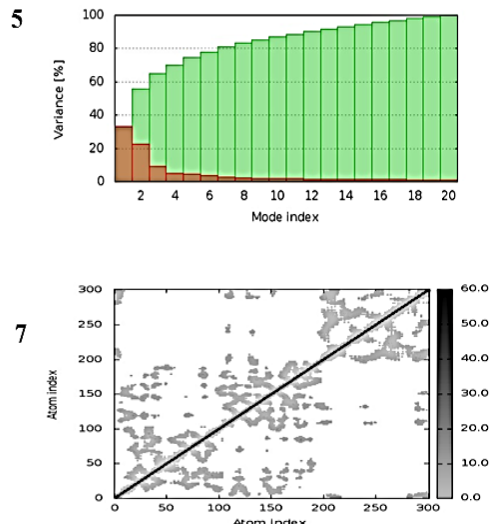

Fig. 4: iMODs normal mode analysis (NMA) of the hit compound PubChem Id 60838 with the targeted receptor

IMODs also measure the B-factor and structural deformity and calculate the eigenvalue. Image 1 represents the docked complex of our protein and ligand. Image 2 of the fig. represents the deformability graph. The deformity graph illustrated peaks in the graph, which represent regions in the protein with deformability. Image 3 represents the B-Factor graph. The main-chain deformability, also known as the B-Factor, is a measure of a molecule's ability to deform at each of its residues. Image 4 represents the eigenvalue of the complex. The motion stiffness is represented by the eigenvalue associated with each normal mode. Its value is proportional to the amount of energy required to distort the structure. The simpler the deformation, the lower the eigenvalue. Our docked complex demonstrated an eigenvalue of 1.4141084e-04. Image 5 represents the variance plot. The variance plot demonstrates individual variances in red color, whereas cumulative variance in green color. Image 6 represents the covariance map. This map demonstrates the correlation motion between a pair of residues in red color, uncorrelated motion in white color, and anti-correlated motion in blue color. Image 7 represents the elastic map of our docked complex. Each dot in the graph represents one spring inside the atoms' pair. The dots are colored dependent on stiffness, with darker grey dots indicating stiffer springs and lighter grey dots indicating softer springs. From the molecular dynamics study, it was evident that our complex showed a good amount of deformability. Furthermore, it also showed a moderately low eigenvalue, suggesting that it could be deformed easily. The variance map exhibited a higher degree of cumulative variances than an individual variance. The elastic network map also produced satisfactory results.

For MD Simulation using the Desmond program for our hit compoundreceptor complex, the protein RMSD fluctuated between 2-18 ns but then showed a stable trajectory up to $50 \mathrm{~ns}$. The ligand RMSD exhibited fluctuations from $0-12$ ns but then demonstrated a stable trajectory up to $50 \mathrm{~ns}$ (fig. 5). Regarding the Protein RMSF analysis, the highest fluctuation was observed at $4.2 \AA$. Overall, the Protein and Ligand RMSF trajectories were found to be stable (fig. 6, fig. 7). Other than these, the amino acid interactions of our protein-ligand complex were also analyzed. The notable hydrogen interactions were observed at PR052, ARG188. The notable hydrophobic interactions were observed at ARG40, ILE43, TYR54, LEU57, LEU58, HIS80, MET82, ARG188. Water bridges were observed in ARG40, ASP48, MET49, ASN51, PR052, TYR54, MET82, ASP187, ARG188, GLN199. Among these residues, only TYR54 and HIS80 exhibited strong interactions throughout the entire simulation process (fig. 8, fig. 9, fig. 10). Other than this, other ligand properties of the hit compound such as radius of gyration, molecular surface area, intramolecular hydrogen bonds, solvent accessible surface area, and polar surface area were monitored throughout the $50 \mathrm{~ns}$ simulation process (fig. 11). The radius of gyration, molecular surface area, and polar surface area plots of the hit compound demonstrated stable trajectories throughout the entire 50 ns simulation study. The solvent-accessible surface area plot showed slight fluctuations up to 15 ns but still showed a stable trajectory throughout the rest of the simulation process. Furthermore, the intramolecular hydrogen bond plot of the hit compounds demonstrated zero hydrogen bonds.

\section{Protein-Ligand RMSD}

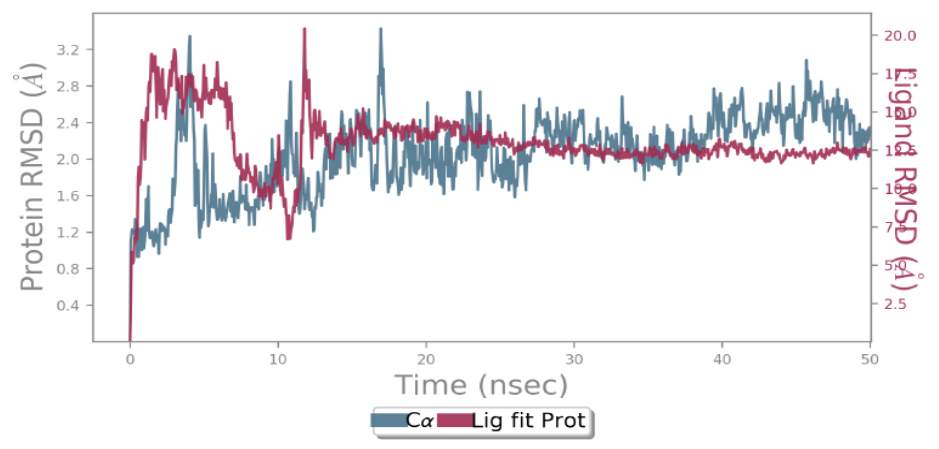

Fig. 5: Protein-ligand RMSD plot 


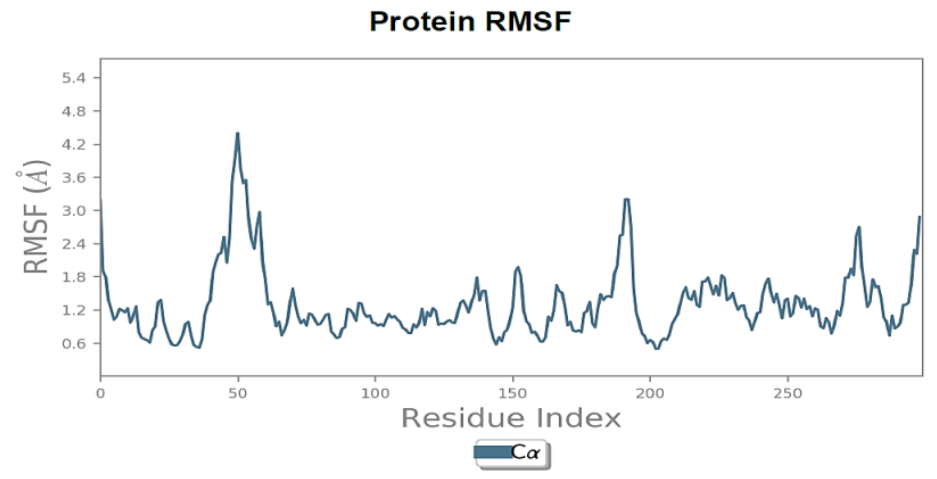

Fig. 6: Protein RMSF plot

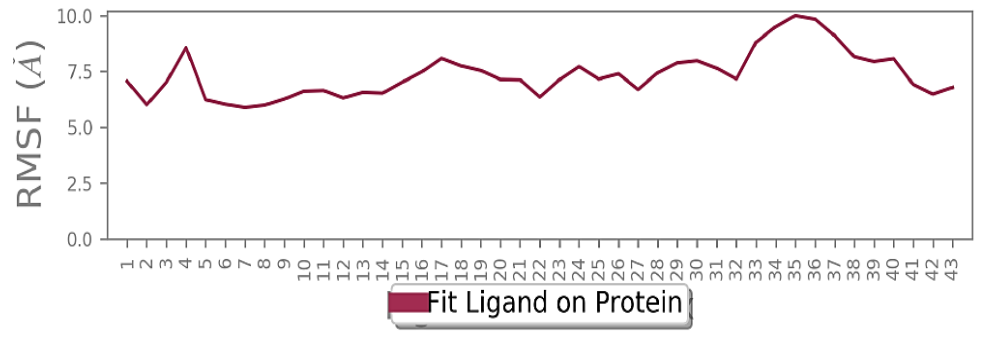

Fig. 7: Ligand RMSF plot

Protein-Ligand Contacts

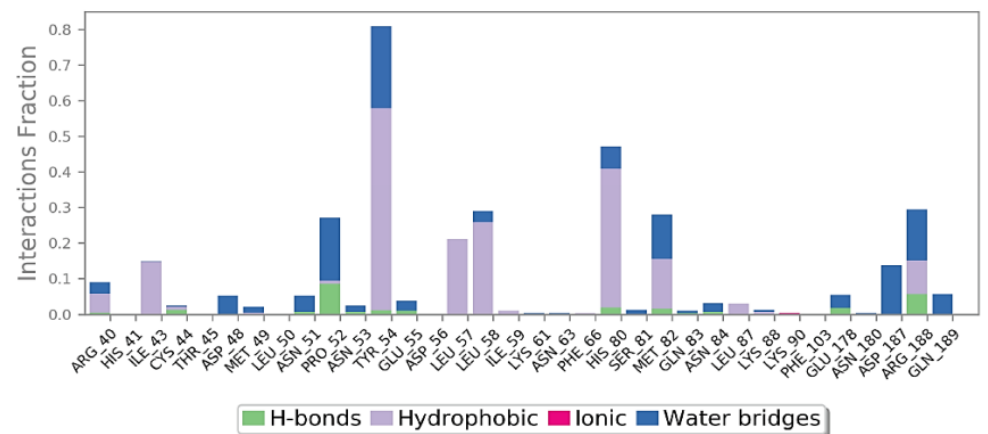

Fig. 8: Protein-Ligand contacts plot detailing amino acid interactions concerning interaction fraction

\section{Ligand-Protein Contacts}
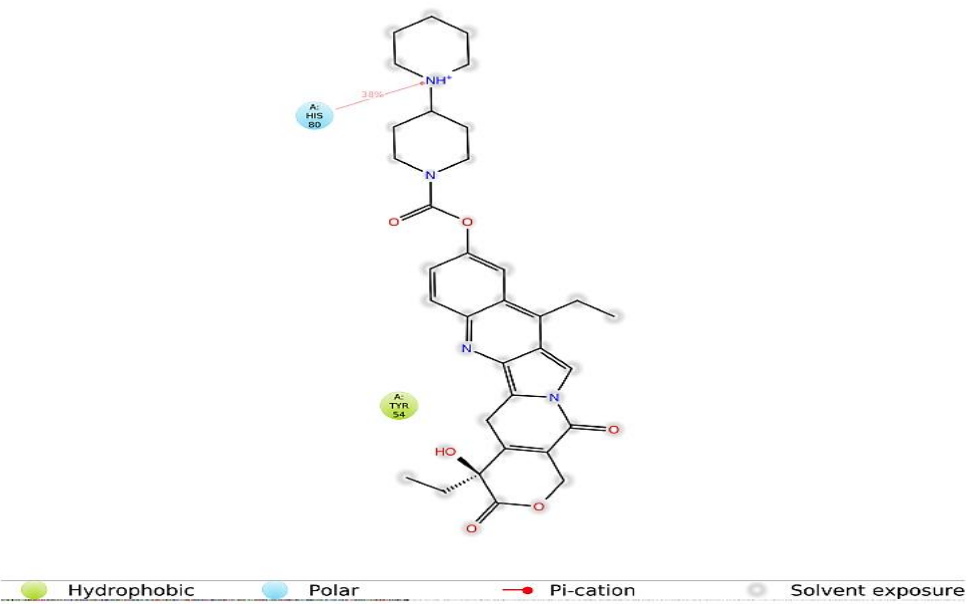

Fig. 9: Ligand-protein contact detailing best prominent amino acid interactions during the simulation process 
Protein-Ligand Contacts (cont.)
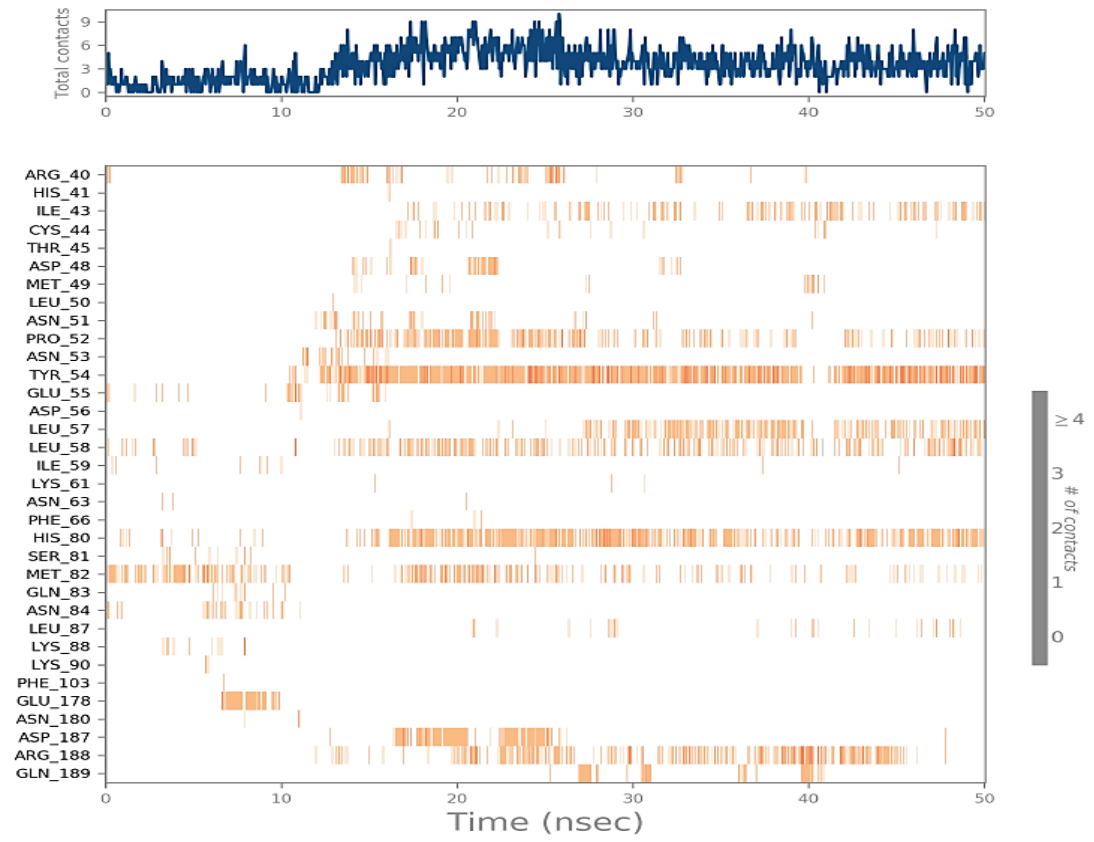

Fig. 10: Protein-ligand contacts plot detailing amino acid interactions concerning the time

Ligand Properties

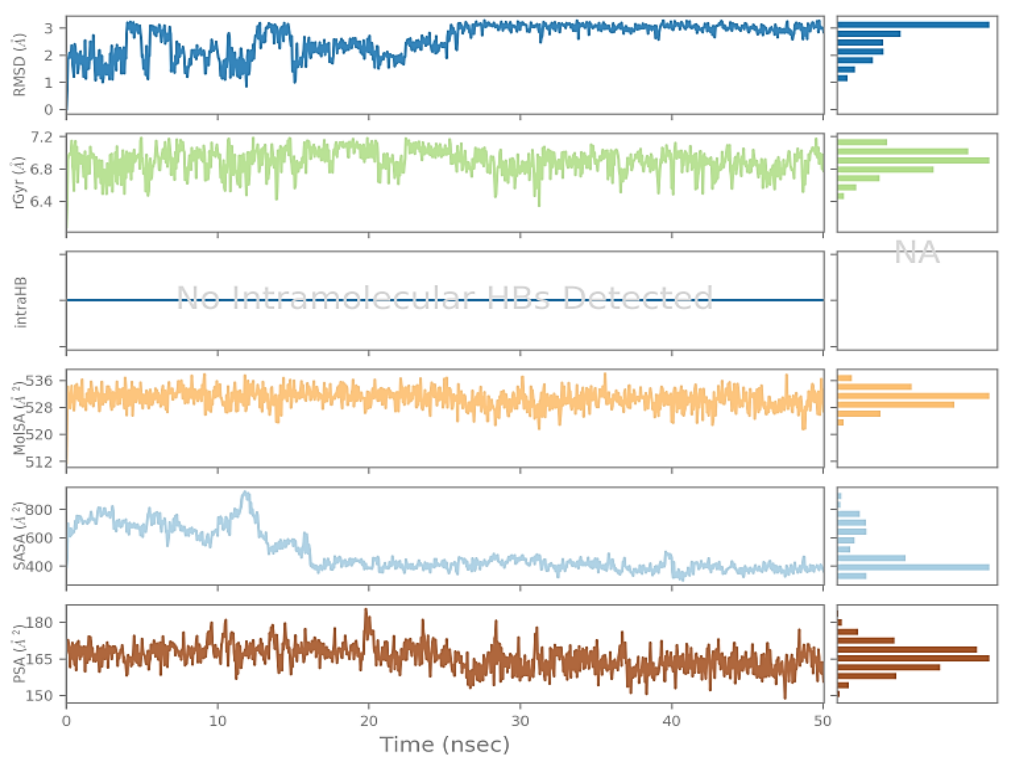

Fig. 11: Root mean square deviation, radius of gyration, intramolecular hydrogen bonds, molecular surface area, solvent accessible surface area, polar surface area plots of the hit compound

\section{DISCUSSION}

SARS CoV-2 3CLpro, the main target chosen in this study, is a multifunctional protein involved in viral RNA transcription and replication. It also includes proteinases, which are responsible for polyprotein cleavage. By interacting with the 40s ribosomal subunit, it also suppresses host translation. The 3C like protease (3CLpro) hydrolyzes polyproteins to create functional proteins. It is required for coronavirus replication and is seen as an important therapeutic target for coronavirus disorders, particularly coronavirus disease 2019. (COVID-19). During coronavirus replication, the 3CLpro cysteine protease hydrolyzes the polyproteins $\mathrm{pp} 1 \mathrm{a}$ and $\mathrm{pp} 1 \mathrm{~b}$ to create functional proteins. Because of its highly conserved sequence and crucial functional features, 3CLpro has been validated as a viable target for the development of medicines to treat SARS, MARS, and COVID-19 [16].

There are currently no officially recognized or authorized targeted treatment agents or medications to treat the viral infection caused by SARS-CoV-2. Effective treatment techniques are still few, and the current standard of care is supportive care. Antiviral therapeutics that target critical proteins involved in the SARS-CoV life cycle would be ideal. Antiviral treatments (such as remdesivir, favipiravir, and lopinavir/ritonavir) have been proposed for the treatment of COVID19 , but their efficacy has not been fully demonstrated, and toxicity issues must be examined and handled systematically and 
comprehensively. Small-molecule medications approved for other human diseases may limit the virus-host interaction of novel CoVs, but much more research is needed to develop specific anti-CoV agents for preventative and treatments, as well as related molecular pathways surrounding viral infections [17].

Therapeutic candidates are mostly focused on major protease inhibitors, ACE2 inhibitors, viral RNA polymerase inhibitors, and prospective drug candidates to prevent SARS-CoV-2 spike glycoprotein trimerization. Furthermore, combination therapy may be required and has high efficacy against SARS-CoV-2. Currently, scientists and researchers are attempting to identify potential drug targets and related mechanistic aspects that can aid in the development of novel therapeutics for this infectious virus; several preclinical studies have suggested various FDA-approved drugs for clinical trials, and importantly, the administration of these drugs can be associated with severe adverse side effects due to Innovative targeted drug delivery systems (multifunctionalization for specific tissue/organ targeting), nano-based structures, metal-grafted graphene oxide, nanocomposites, nano-phytotherapeutics, biodegradable nanocarriers, carbon nanotubes, and multidrug nanoparticles can all contribute to the fight against the SARS-CoV-2 virus. The aim of this current study was to generate suitable drug candidates from previously used nanoparticles as well as nanocarrier drug delivery systems to counter the 3CLpro protein of SARS CoV-2 with the help of virtual screening and molecular docking techniques $[18,19]$.

\section{CONCLUSION}

In the current investigation, VS and molecular docking analysis results were found to be useful strategies for discovering inhibitors of the SARS COV-2 3CL Protease. According to the findings, the two final hit compounds investigated, PubChem Id 58823276 and PubChem Id 60838, had a high affinity for the 3CL pro binding region of SARS COV-2 (fig. 2, fig. 3). Their binding affinity was determined to be- $9.6 \mathrm{~kJ} / \mathrm{mol}$ and $-8.5 \mathrm{~kJ} / \mathrm{mol}$, respectively. The two final compounds were found to be tightly attached to the $3 \mathrm{CL}$ protease of SARS COV-2, showing that they were more strongly coupled than the co-crystal ligand. The best docking results all passed the Lipinski rule of five, indicating that they are likely to be medicinally active pharmaceuticals. The results revealed that the compounds interacted with amino acids more closely than the native ligand. The use of VS and molecular docking approaches may significantly lower the cost of drug development and production, and as a result, provided evidence for previously undiscovered interactions between the identified chemical and the target SARS COV-2 3CL protease. Experiments (in vivo) are needed to confirm the findings and evaluate the impact of the chemicals on COVID-19 using an appropriate animal model.

\section{ACKNOWLEDGMENT}

We thank BioNome for providing excellent insight regarding Bioinformatics Skills.

\section{FUNDING}

Nil

\section{AUTHORS CONTRIBUTIONS}

All authors have contributed equally.

\section{CONFLICT OF INTERESTS}

Declared none

\section{REFERENCES}

1. Biju P, KP, Revadigar V, Dsouza S, Asif Iqbal M, Ahmed G. A review on the impact of the COVID-19 pandemic on the health care sector. Int J Pharm Pharm Sci. 2021;13(10):1-6. doi: 10.22159/ijpps.2021v13i10.42566.
2. Jamalipour Soufi G, Iravani S. Potential inhibitors of SARS-CoV2: recent advances. J Drug Target. 2021;29(4):349-64. doi: 10.1080/1061186X.2020.1853736, PMID 33210953.

3. Rathnayake AD, Zheng J, Kim Y, Perera KD, Mackin S, Meyerholz DK, Kashipathy MM, Battaile KP, Lovell S, Perlman S, Groutas WC, Chang KO. 3C-like protease inhibitors block coronavirus replication in vitro and improve survival in MERS-CoV-infected mice. Sci Transl Med. 2020;12(557):1-16. doi: 10.1126/scitranslmed.abc5332, PMID 32747425.

4. Su H, Yao S, Zhao W, Zhang Y, Liu J, Shao Q, Wang Q, Li M, Xie H, Shang W, Ke C, Feng L, Jiang X, Shen J, Xiao G, Jiang H, Zhang L, Ye Y, Xu Y. Identification of pyrogallol as a warhead in the design of covalent inhibitors for the SARS-CoV-2 3CL protease. Nat Commun. 2021;12(1). Available from: http://dx:3623. doi: 10.1038/s41467-021-23751-3, PMID 34131140.

5. Kontoyianni M. Docking and virtual screening in drug discovery. Methods Mol Biol. 2017;1647:255-66. doi: 10.1007/978-1-4939-7201-2_18, PMID 28809009.

6. Kim S, Thiessen PA, Bolton EE, Chen J, Fu G, Gindulyte A, Han L, He J, He S, Shoemaker BA, Wang J, Yu B, Zhang J, Bryant SH. PubChem substance and compound databases. Nucleic Acids Res. 2016;44(D1):D1202-13. doi: 10.1093/nar/gkv951, PMID 26400175.

7. Berman HM, Westbrook J, Feng Z, Gilliland G, Bhat TN, Weissig $\mathrm{H}$, Shindyalov IN, Bourne PE. The protein data bank. Nucleic Acids Res. 2000;28(1):235-42. doi: 10.1093/nar/28.1.235, PMID 10592235.

8. Trott 0 , Olson AJ. AutoDock Vina: improving the speed and accuracy of docking with a new scoring function, efficient optimization, and multithreading. J Comput Chem. 2010;31(2):455-61. doi: 10.1002/jcc.21334, PMID 19499576.

9. Visualizer. In: Accelrys Software Inc. D Sv4. Vol. 0(100). 13345; 2005.

10. Client DS, Studio D. Discovery T, Client S. Introduction discov studio client; 2020. p. 1-7.

11. Daina A, Michielin 0, Zoete V. SwissADME: A free web tool to evaluate pharmacokinetics, drug-likeness and medicinal chemistry friendliness of small molecules. Sci Rep. 2017;7(October):42717. doi: 10.1038/srep42717, PMID 28256516.

12. Lee S, Chang G, Lee I, Chung J, Sung K, No K. The PreADME: pcbased program for the batch prediction of ADME properties. EuroQSAR; 2004.p. 9.

13. Molinspiration cheminformatics. Choice. Rev Online. 2006;43(11).

14. Bhowmik R, Roy S, Sengupta S, Sharma S. Biocomputational and pharmacological analysis of phytochemicals from Zingiber officinale (Ginger), Allium sativum (Garlic), and Murrayakoenigii (Curry. Zingiber officinale (Ginger), Allium sativum (Garlic), and Murrayakoenigii (Curry Leaf). Int J Appl Pharm. 2021;13(5):280-6.

15. Daina A, Zoete V. A BOILED-egg to predict gastrointestinal absorption and brain penetration of small molecules. Chem Med Chem. 2016;11(11):1117-21. doi: 10.1002/ cmdc.201600182, PMID 27218427.

16. Kwatra B, Roy R, Bhowmik R, Sengupta S. Drug repurposing: in silico modeling of COVID-19. Res J Life Sci Bioinformatics Pharm Chem Sci. 2021;7(2):19-40.

17. Singh DD, Han I, Choi EH, Yadav DK. Recent advances in pathophysiology, drug development and future perspectives of SARS-CoV-2. Front Cell Dev Biol. 2020;8(1124):580202. doi: 10.3389/fcell.2020.580202, PMID 33240881.

18. Shaji J, Shaikh M. Drug-resistant tuberculosis: recent approach in polymer-based nanomedicine. Int J Pharm Pharm Sci. 2016;8(10):1-6. doi: 10.22159/ijpps.2016v8i10.11295.

19. Nath R, Roy R, Mondal B, Bhattacharya B, Ravi L. Nanoparticle's significance as antibacterial agents and other pharmaceutical applications and their limitations: A critical review. J Pharm Res Int. 2021;33(38B):8-27. doi: 10.9734/jpri/ 2021/ v33i38B32093. 